\title{
Data on the Lumbricidae and Enchytraeidae from six forest sites in Germany and Portugal
}

\author{
J. RÖMBKE ${ }^{1 *}$, C. BANDOW ${ }^{1}$, A. GERLACH ${ }^{3}$, B. JAESCHKE ${ }^{3}$, R. M. SCHMELZ ${ }^{1,2}$, T. NATAL-DA-LUZ ${ }^{4}$, \\ J.P. SOUSA ${ }^{4}$ \& D.J. RUSSELL ${ }^{3}$ \\ ${ }^{1}$ Jörg Römbke \& Cornelia Bandow, ECT Oekotoxikologie GmbH, Flörsheim/Main, Germany. \\ *Corresponding author: J-roembke@ect.de \\ ${ }^{2}$ Rüdiger M. Schmelz, ECT Oekotoxikologie GmbH, Flörsheim/Main, Germany and University of A \\ Coruña, Science Faculty, Evolutionary Biology Research Group (GIBE), A Coruña, Spain. \\ ${ }^{3}$ Andreas Gerlach \& Barbara Jaeschke \& David J. Russell, Senckenberg Museum of Natural History \\ Görlitz, Germany. \\ ${ }^{4}$ Tiago Natal-Da-Luz \& Jose Paulo Sousa, Centre for Functional Ecology,Department of Life Sciences, \\ University of Coimbra, Portugal.
}

\begin{abstract}
The complex interactions between primary production, soil and soil organisms (in particular Lumbricidae and Enchytraeidae) during organic matter decomposition have developed over evolutionary time under specific habitat conditions. Thus, in order to study these processes, four forest sites in Germany (two in southern Hesse, two in eastern Saxony) and two sites in Central Portugal were selected to study both the oligochaete fauna and litter decomposition (almost all of them with a $\mathrm{pH}<4$ ). Here, the results of the first part of this his work, i.e. the abundance and the species composition of the oligochaete fauna, are described. All samplings were performed using standard ISO-methods, with different temporal intensity. In detail, nine common Central European earthworm species were found at the six study sites plus one unidentified species at a Portuguese site. Abundance was usually low $\left(<10 \mathrm{Ind} / \mathrm{m}^{2}\right)$; higher numbers $\left(72.5\right.$ and $\left.17.5 \mathrm{Ind} / \mathrm{m}^{2}\right)$ were detected only at the two Saxonian sites. In total, 53 enchytraeid species were found; 43 of them already described. At four sites densities fell within the expected range for German deciduous forests $\left(20000-80000 \mathrm{Ind} / \mathrm{m}^{2}\right)$. Finally, notes on the taxonomy and biogeography of several potworm species are given.
\end{abstract}

Keywords: Annelida, species number, species composition, global climate change

\section{INTRODUCTION}

\begin{abstract}
A ccording to recent research on climatechange scenarios, Central European weather will become more extreme, with intermittent very hot and dry summers during the next 50-100 years (Schär et al. 2004, Schmidli et al. 2007). Tree species currently used in forestry are not adapted to such conditions, thus drought-resistant Mediterranean tree species might be an alternative (Pflug \& Brüggemann 2012, Bussotti et al. 2015). Litterfall from trees represents a key resource for the below-ground ecosystem, but it is not known whether the complex process of litter decomposition, which depends on the soil organism community (mainly bacteria, fungi and a wide range of soil invertebrates), is or will be impacted by these climatically driven changes in forest composition (Blankinship et al. 2011 but see Wall et
\end{abstract}

al. 2008). Soil organism communities are adapted in various ways to local conditions, e.g. soil properties or climatic factors. As part of the German BiK-F project (www. www.bik-f.de; today belonging to the Senckenberg Museum, Frankfurt), the question was raised whether climate-change driven transformations of forest-tree composition will affect these organisms and their functions, such as their role in litter decomposition. Specifically, one of the research questions is whether soil organisms in Germany, having been adapted to litter from Central European tree species, such as beech (Fagus sylvatica) and oaks, e.g. Quercus pubescens, can handle litter from southern European trees (mainly oaks). Lumbricid and enchytraeid species were selected as representatives of the whole community due to their important role 
in litter decomposition under central European conditions (Blouin et al. 2013).

The oligochaete studies were performed at different intensities in six forest sites (two in Saxony [eastern Germany], two in Hesse [western Germany] and two in Central Portugal), representing areas with different climate systems. Thus, the main question of the whole project was: will the local soil organism community be able to decompose "foreign" litter, or will local soil nutrient cycles be disrupted after introduction of the "foreign" trees? In this paper we report on the description of the oligochaete fauna at the six study sites in Germany and Portugal. Abundance and community composition of the fauna are important for the interpretations of the litter decomposition studies which are still in progress.

\section{MATERIAL AND METHODS}

In order to address the research question, three geographical regions in Europe were selected, one in the continental and one in the Atlantic region of Germany, and one in the Mediterranean region (Central Portugal). The study sites in eastern Germany are located in the continental climate zone with a mean annual yearly temperature (MAT) average of $8.5^{\circ} \mathrm{C}$. These average increases by approx. $2^{\circ} \mathrm{C}$ when shifting to the sites in western Germany, representing the oceanic climate zone. An additional further $3{ }^{\circ} \mathrm{C}$ increase in yearly temperature MAT is found at the study sites in Portugal which are influenced by the Mediterranean climate zone (Peel et al. 2007). These differences are within the range of predicted temperature increases for Germany and Portugal.

In each region, two study sites with four $20 \times 20$ $\mathrm{m}$ plots each were selected in order to represent different forest histories and conditions within a climatic region, i.e. forests were only replicated within sites but not necessarily between sites of an area. The two eastern German sites are located in the state of Saxony: Viereichen and Ullersdorf, both near Görlitz. They represent two undisturbed deciduous forests, mainly consisting of different species of oaks (mainly Quercus robur with an age of about 100 years), hornbeam (Carpinus betulus) and few common beech (Fagus sylvatica) trees. At Viereichen, rarely small leaf linden (Tilia cordata) does occur.

The two south-western German sites are located in the state of Hesse: Rüsselsheim (disturbed mixed forest near human settlements) and Lampertsheim (mixed deciduous forest in the Rhine valley, very sandy soil and dry conditions). These two were most intensively studied. The Rüsselsheim site is originally an open Pinus sylvestris forest containing solitary old ( $>80$ years) Quercus robur trees. After disturbed by fire in 2008, the area was partly re-planted with $Q$. ilex, $Q$. frainetto and $Q$. pubescens. At Lampertheim, the dominant tree species is also Q. robur (ca. 110 years old). Saplings of the same oak species as in Rüsselsheim (plus $Q$. petraea) where planted just outside of the undisturbed sampling area (Dorow et al. 2012). St. Olaia is a calcareous hill in the coastal plain that has been continuously occupied by forest since the Neolithic times. The vegetation is mainly composed by the tree species Quercus fagineae and Quercion ilicis and various herb and shrub species (e.g. Vinca difformis). Cerdeira has been occupied by forest since about 50 years ago when the area was no longer explored for agriculture. The vegetation is mainly composed by the tree species Quercus robur, Q. pyrenaica, Castanea sativa, Pinus pinaster and Eucalyptus globulus and the bracken fern (Pteridium aquilinum).

The main characteristics of the six study sites and their most important soil properties are given in Table 1. Soils differ strongly in terms of texture, mainly regarding the percentage of sand and silt. The clay content differs only slightly (i.e. between 5 and 10\%). Except for St. Olaia, which had a $\mathrm{pH}$ of 5.0, all other sites had acid soils with $\mathrm{pH}$-values between 3.2 and 3.7. Regarding the organic matter content, a wide range was covered, from $6.2 \%$ in Lampertheim up to $21.3 \%$ at Cerdeira. Despite these differences, the $\mathrm{C} / \mathrm{N}$-ratio was not strikingly different, ranging from 13.1 in Ullersdorf and 20.3 in Rüsselsheim. Soil moisture was measured gravimetrically (i.e. loss in \% weight after drying at $105^{\circ} \mathrm{C}$ ) in parallel to the individual oligochaete samplings. It can change quickly, but the two Hessian sites are certainly drier than the other four sites. 
Römbke et al.: Data on the Lumbricidae and Enchytraeidae from six forest sites in Germany and Portugal

Table 1. Main characteristics and most important soil properties of the six study sites. Mean temperature $=$ mean of daily measures during the study period. Mean annual precipitation $(\mathrm{mm} / \mathrm{year})$ data are long-term averages collected from the nearest climate station.

\begin{tabular}{|c|c|c|c|c|c|c|}
\hline Property & Viereichen & Ullersdorf & Rüsselsheim & Lampertheim & Cerdeira & St. Olaia \\
\hline Region & \multicolumn{2}{|c|}{ Saxony (Germany) } & \multicolumn{2}{|c|}{ Hesse (Germany) } & \multicolumn{2}{|c|}{ Central Portugal } \\
\hline Coordinates & $\begin{array}{l}51^{\circ} 22^{\prime} 54^{\prime \prime} \mathrm{N} \\
14^{\circ} 41^{\prime} 54^{\prime \prime} \mathrm{E}\end{array}$ & $\begin{array}{l}51^{\circ} 14^{\prime} 15^{\prime \prime} \mathrm{N} \\
14^{\circ} 50^{\prime} 27^{\prime \prime} \mathrm{E}\end{array}$ & $\begin{array}{c}49^{\circ} 57^{\prime} 13^{\prime \prime} \mathrm{N} \\
8^{\circ} 24^{\prime} 54^{\prime \prime} \mathrm{E}\end{array}$ & $\begin{array}{c}49^{\circ} 35^{\prime} 08^{\prime \prime} \\
\mathrm{N} \\
8^{\circ} 28^{\prime} 46^{\prime \prime} \mathrm{E}\end{array}$ & $\begin{array}{l}40^{\circ} 09^{\prime} 43^{\prime \prime} \mathrm{N} \\
8^{\circ} 19^{\prime} 41^{\prime \prime} \mathrm{W}\end{array}$ & $\begin{array}{l}40^{\circ} 17^{\prime} 06^{\prime \prime} \mathrm{N} \\
8^{\circ} 71^{\prime} 52^{\prime \prime} \mathrm{W}\end{array}$ \\
\hline Text. Sand (\%) & 62.3 & 19.5 & 67.8 & 75.4 & 35.1 & 48.9 \\
\hline Text. Silt (\%) & 32.2 & 70.2 & 21.9 & 19.0 & 57.2 & 43.0 \\
\hline Text. Clay (\%) & 5.5 & 10.3 & 10.3 & 5.6 & 7.7 & 8.1 \\
\hline Texture class & $\begin{array}{l}\text { Medium silty } \\
\text { sand }\end{array}$ & $\begin{array}{l}\text { Weakly loamy } \\
\text { silt }\end{array}$ & $\begin{array}{l}\text { Medium } \\
\text { loamy sand }\end{array}$ & $\begin{array}{l}\text { Weakly } \\
\text { silty sand }\end{array}$ & Sandy silt & $\begin{array}{l}\text { Silty-loamy } \\
\text { sand }\end{array}$ \\
\hline $\mathrm{pH}\left(\mathrm{CaCl}_{2}\right)$ & 3.7 & 3.2 & 3.5 & 3.6 & 3.5 & 5.0 \\
\hline $\begin{array}{l}\text { Organic Material } \\
(\%)\end{array}$ & 9.5 & 13.4 & 9.4 & 6.2 & 21.3 & 16.7 \\
\hline $\mathrm{C} / \mathrm{N}$ & 14.9 & 13.1 & 20.3 & 17.1 & 15.7 & 13.0 \\
\hline $\begin{array}{l}\text { Ø Soil Moisture } \\
(\%)\end{array}$ & 30.1 & 79.5 & 37.8 & 30.9 & 41.8 & 30.6 \\
\hline $\begin{array}{l}\text { O Temperature } \\
\left(\mathrm{C}^{\circ}\right)\end{array}$ & 8.4 & 9.9 & 11.1 & 7.3 & 12.8 & 15.1 \\
\hline $\begin{array}{l}\text { O Ann. } \\
\text { Precipitation }\end{array}$ & 672 & 635 & 658 & 695 & 958 & 900 \\
\hline
\end{tabular}

\section{Sampling of soil organisms}

Earthworms (Lumbricidae) were sampled according to the ISO standard 23611-1 (2006), i.e. by a combination of hand-sorting and formalin extraction. Five randomly selected samples were taken per plot. The two Hessian sites were sampled 2009, twice in spring and autumn each. The remaining four sites (two in Saxony and two in Portugal) were sampled once in April and June 2014, respectively. Each individual worm was morphologically determined using the keys of Graff (1953), Bouché (1972) and Sims \& Gerard (1999). The worms were stored in $90 \%$ ethanol.
Potworms (Enchytraeidae) were sampled according to the ISO standard 23611-3 (2006) with soil-corers $(\varnothing 5.5 \mathrm{~cm})$. Ten samples were randomly taken per plot. Each sample was divided to litter layer and $0-4 \mathrm{~cm}$ mineral soil layer. The Hessian sites were sampled four times between autumn 2008 and spring 2010, while the others were sampled only once, in April or June 2014. Potworms were collected by wet extraction in the laboratory at room temperature, about $20^{\circ} \mathrm{C}$. Extraction duration for litter-layer samples was 1-2 days and for mineral soil samples was 3-4 days. Morphological identification was performed using the key and checklist of Schmelz \& Collado (2010; 2012). 


\section{RESULTS}

\section{Abundance, species number and composition of earthworms}

All three pairs of sites and, also, the two sites within a region differed considerably in abundance and/or species number (Table 2). In Hesse, very low density $\left(0.4-3.4 \mathrm{Ind} / \mathrm{m}^{2}\right)$ and species richness were found. In Saxony we found considerable differences between the two sites. At Viereichen both abundance and species number were high $\left(80.5 \mathrm{Ind} / \mathrm{m}^{2} ; 6\right.$ species), but at Ullersdorf the respective numbers were clearly lower (17.5 Ind $/ \mathrm{m}^{2} ; 3$ species). In Portugal, the differences were even more pronounced; at
Cerdeira almost no earthworms were found $(0.4$ $\mathrm{Ind} / \mathrm{m}^{2} ; 1$ species), while at St. Olaia both parameters were much higher (7.9 Ind $/ \mathrm{m}^{2} ; 4$ species).

\section{Abundance, species number and composition of enchytraeids}

Due to the different sampling efforts at the six sites, it is difficult to compare the results directly, but the number of enchytraeids was in tendency, with two exceptions, in a similar range, i.e. about 40000 to $50000 \mathrm{Ind} / \mathrm{m}^{2}$ (Tables 3 and 4). In Rüsselsheim their average number was almost always lower (about $25000 \mathrm{Ind} / \mathrm{m}^{2}$ ), while at Viereichen on average twice as many enchytraeids were found as at all other sites.

Table 2. Abundance $\left(\mathrm{Ind} / \mathrm{m}^{2}\right)$, standard deviation (SD), species number and composition of earthworms at the six study sites. Note that earthworms were sampled four times at the two Hessian sites, but only once at the other sites (therefore, mean values are given for Rüsselsheim and Lampertheim). n.d. = not determined due to low numbers

\begin{tabular}{|c|c|c|c|c|c|c|c|c|}
\hline \multirow[t]{2}{*}{ Genus / Species } & \multirow{2}{*}{$\begin{array}{c}\text { Vieleichen } \\
\text { Spring } \\
2014\end{array}$} & \multirow{2}{*}{$\begin{array}{c}\text { Ullersdorf } \\
\text { Spring } \\
2014\end{array}$} & \multicolumn{2}{|c|}{ Rüsselsheim } & \multicolumn{2}{|c|}{ Lampertheim } & \multirow{2}{*}{$\begin{array}{c}\text { Cerdeira } \\
\text { Spring } \\
2014\end{array}$} & \multirow{2}{*}{$\begin{array}{c}\text { St. Olaia } \\
\text { Spring } \\
2014\end{array}$} \\
\hline & & & $\begin{array}{l}\text { Autumn } \\
2009\end{array}$ & $\begin{array}{l}\text { Spring } \\
2010\end{array}$ & $\begin{array}{l}\text { Autumn } \\
2009\end{array}$ & $\begin{array}{l}\text { Spring } \\
2010\end{array}$ & & \\
\hline Aporrectodea s.l. sp. & 23.0 & 0.5 & - & - & - & - & - & 2.5 \\
\hline $\begin{array}{l}\text { Aporrectodea caliginosa } \\
\text { (Savigny, 1826) }\end{array}$ & 14.5 & - & - & - & - & - & - & - \\
\hline $\begin{array}{l}\text { Aporrectodea rosea } \\
\text { (Savigny, 1826) }\end{array}$ & 0.5 & 0.5 & - & - & - & - & - & 3.0 \\
\hline Lumbricus sp. & 12.0 & 4.5 & 2.0 & - & 1.3 & - & - & - \\
\hline $\begin{array}{l}\text { Lumbricus rubellus } \\
\text { Hoffmeister, } 1843 \\
\end{array}$ & 14.0 & 3.5 & 0.8 & 1.2 & 1.3 & 0.4 & 0.4 & - \\
\hline $\begin{array}{l}\text { Lumbricus terrestris } \\
\text { Linnaeus, } 1758\end{array}$ & 1.5 & - & - & - & - & - & - & - \\
\hline Dendrobaena/-drilus sp. & 2.5 & 0.5 & - & - & - & - & - & - \\
\hline $\begin{array}{l}\text { Dendrobaena attemsi } \\
\text { (Michaelsen, 1902) }\end{array}$ & 4.0 & 5.0 & - & - & - & - & - & - \\
\hline $\begin{array}{l}\text { Dendrobaena octaedra } \\
\text { (Savigny, 1826) }\end{array}$ & - & - & - & - & 0.5 & - & - & - \\
\hline $\begin{array}{l}\text { Dendrodrilus rubidus } \\
\text { (Savigny, 1826) }\end{array}$ & 0.5 & - & - & - & - & - & - & - \\
\hline Octolasion sp. & - & - & - & - & - & - & - & 0.5 \\
\hline $\begin{array}{l}\text { Octolasion cyaneum } \\
\text { (Savigny, 1826) }\end{array}$ & - & - & - & - & - & - & - & 1.5 \\
\hline $\begin{array}{l}\text { Octolasion tyrtaeum } \\
\text { (Savigny, 1826) }\end{array}$ & - & - & - & - & 0.3 & - & - & - \\
\hline Unknown species & - & - & - & - & - & - & - & 0.5 \\
\hline Undetermined & 8.0 & 3.0 & 0.5 & - & - & - & - & - \\
\hline Total \pm SD & $\begin{array}{c}80.5 \\
\pm 32.2 \\
\end{array}$ & $\begin{array}{r}17.5 \\
\pm 3.4 \\
\end{array}$ & $\begin{array}{c}3.3 \\
\pm 1.7 \\
\end{array}$ & $\begin{array}{l}1.2 \\
\text { n.d. }\end{array}$ & $\begin{array}{r}3.4 \\
\pm 2.1 \\
\end{array}$ & $\begin{array}{l}0.4 \\
\text { n.d. }\end{array}$ & $\begin{array}{l}0.4 \\
\text { n.d. }\end{array}$ & $\begin{array}{c}8.0 \\
\pm 2.0 \\
\end{array}$ \\
\hline Species number per site & 6 & 3 & 1 & 1 & 3 & 1 & 1 & 4 \\
\hline
\end{tabular}


Table 3. Abundance $\left(\mathrm{Ind} / \mathrm{m}^{2}\right)$ and standard deviation (SD) of enchytraeids at Rüsselsheim and Lampertheim

\begin{tabular}{|l|l|c|c|}
\hline & & \multicolumn{2}{|c|}{ Hesse (Germany) } \\
\hline Year & Season & Rüsselsheim & Lampertheim \\
\hline 2008 & Autumn & $21520 \pm 3475$ & $57840 \pm 8286$ \\
\hline 2009 & Spring & $41940 \pm 5841$ & $13060 \pm 715$ \\
\hline 2009 & Autumn & $13920 \pm 2994$ & $48440 \pm 12517$ \\
\hline 2010 & Spring & $22700 \pm 7569$ & $\mathbf{4 8 7 3 5} \pm \mathbf{2 6 ~ 3 2 4}$ \\
\hline Average & - & $\mathbf{2 5 0 2 0} \pm \mathbf{1 1 2 3 2}$ & \\
\hline
\end{tabular}

Enchytraeid abundance was fairly similar at the two Portuguese sites. At the two Hessian sites, samples were taken four times between the autumn of 2006 and the spring of 2011. Variation in abundance were less pronounced in Rüsselsheim (variation of a factor of three) than in Lampertheim (variation of a factor of six), but no specific pattern was visible.

In total, 53 enchytraeid species were identified (Table 4). Forty-three of them have already been described or are known to science; the remaining ten require descriptions. Not surprisingly, all but one of the undescribed taxa were found at the two Portuguese sites. Enchytraeids of Portugal are poorly known (Schmelz \& Collado 2013). The number of enchytraeid species per site varied between 11 (Cerdeira) and 27 (St. Olaia); species numbers at the German sites were in-between. Thirty and 31 species, respectively, were only found at the sites in Germany (four sites) or in Portugal (two sites). Eleven species out of 52 occurred both in Portugal and Germany. Only one species, Enchytronia parva, was found at all six sites. At most sites no (Rüsselsheim) or few, i.e. 1-4 (Lampertheim, Viereichen, Ullersdorf, and Cerdeira) species occurred which were not found at other sites. The exception is St. Olaia, where 20 species were found which were not found at other sites.

\section{DISCUSSION}

\section{Abundance, species number and composition of earthworms}

All but one of the earthworm species found are well-known throughout Europe, i.e. no species with a restricted regional distribution was de- tected. The exception was one small worm found at St. Olaia, which was not well enough preserved to be determined. It resembled Proctodrilus antipai, a central-European species occurring between central France and the Black Sea (Bouché 1972, Csuzdi \& Zicsi 2003).

The low abundances and species numbers at most sites can be explained by the acidic soils with $\mathrm{pH}$-values mainly below four, as indicated by typical acidophilous species of the genera Dendrobaena/Dendrodrilus and, partly, Lumbricus. The low abundance at the Hessian and Portuguese sites can be explained by site-specific characteristics, i.e. very sandy and dry conditions soil in Lampertheim, human disturbance in Rüsselsheim and shallow soil layers, partly on slopes, in Cerdeira. With such low abundances, it is clear that the ecological role of earthworms at these sites is small (cf. Blouin et al. 2013). The exception is Viereichen with a similarly low $\mathrm{pH}$, but with twice the average earthworm densities reported for German deciduous forests (Jänsch et al. 2013). This situation cannot be presently explained.

\section{Abundance, species number and list of enchytraeids}

Enchytraeid densities at the six sites varied between $25000 \mathrm{Ind} / \mathrm{m}^{2}$ (Rüsselsheim) and almost $100000 \mathrm{Ind} / \mathrm{m}^{2}$ (Viereichen). Three out of the four German sites as well as both Portuguese sites are within the "normal" range $(51241 \pm 30677$ $\mathrm{Ind} / \mathrm{m}^{2}$ ) for German deciduous forests, as determined in a recent literature review (Römbke et al. 2013). According to the same source, such German deciduous forests "should" harbor $12.4 \pm 5.5$ 
Table 4. Abundance (Ind $/ \mathrm{m}^{2}$ ), standard deviation (SD.), number and composition of enchytraeid species at the six study sites (mean values for the Hesse sites). Species names with four letter codes in brackets indicate forms with new character combinations, probably new species.

\begin{tabular}{|c|c|c|c|c|c|c|}
\hline \multirow[t]{2}{*}{ Genus / Species } & \multicolumn{2}{|c|}{$\begin{array}{c}\text { Saxony } \\
\text { (Germany) }\end{array}$} & \multicolumn{2}{|c|}{ Hesse (Germany) } & \multicolumn{2}{|c|}{ Portugal } \\
\hline & $\begin{array}{l}\text { Vier- } \\
\text { eichen }\end{array}$ & $\begin{array}{c}\text { Ullers- } \\
\text { dorf }\end{array}$ & $\begin{array}{l}\text { Rüssels- } \\
\text { heim }\end{array}$ & $\begin{array}{c}\text { Lam- } \\
\text { pertheim }\end{array}$ & Cerdeira & St. Olaia \\
\hline Achaeta abulba Graefe, 1989 & - & 80 & 1050 & 10480 & - & - \\
\hline Achaeta affinis Nielsen \& Christensen, 1959 & 22940 & 1000 & 10920 & 16600 & 12960 & - \\
\hline Achaeta bibulba Graefe, 1989 & - & - & 220 & 260 & - & - \\
\hline Achaeta bifollicula Chalupský, 1993 & - & - & 860 & 80 & - & - \\
\hline Achaeta camerani (Cognetti, 1899) & 820 & 11580 & 380 & 1580 & - & - \\
\hline $\begin{array}{l}\text { Achaeta unibulba Graefe, Christensen \& Dózsa- } \\
\text { Farkas, } 2005\end{array}$ & - & - & - & - & - & 60 \\
\hline Achaeta sp. (HEAL) & - & - & - & - & - & 600 \\
\hline Achaeta sp. (PAFF) & - & - & - & - & - & 2680 \\
\hline Achaeta sp. (PAFU) & - & 4560 & - & - & - & - \\
\hline Bryodrilus ehlersi Ude, 1892 & - & - & 10 & 170 & - & - \\
\hline Buchholzia appendiculata (Buchholz, 1862) & 4640 & - & - & - & - & 14840 \\
\hline Buchholzia sp. (OLAI) & - & - & - & - & - & 1080 \\
\hline Cernosvitoviella cf. minor Dózsa-Farkas, 1990 & - & - & - & - & - & 20 \\
\hline Cognettia cognettii (Issel, 1905) & - & 60 & - & 40 & 12200 & - \\
\hline $\begin{array}{l}\text { Chamaedrilus/ Cognettia chlorophilus * Friend, } \\
1913\end{array}$ & 7000 & 17020 & 5410 & 6180 & - & 320 \\
\hline $\begin{array}{l}\text { Enchytraeus bigeminus Nielsen \& Christensen, } \\
1963\end{array}$ & - & - & - & 20 & - & - \\
\hline Enchytraeus buchholzi Vejdovsky, 1879 & 1420 & - & - & - & 540 & 3000 \\
\hline $\begin{array}{l}\text { Enchytraeus bulbosus Nielsen \& Christensen, } \\
1963\end{array}$ & - & - & - & - & - & 240 \\
\hline Enchytraeus sp. (GRAN) & 2020 & - & 10 & 90 & 820 & 1240 \\
\hline Enchytraeus norvegicus Abrahamsen, 1969 & 11020 & 120 & 70 & 165 & 1760 & - \\
\hline Enchytronia parva Nielsen \& Christensen, 1959 & 8440 & 180 & 340 & 2090 & 1500 & 840 \\
\hline Enchytronia sp. (MINO) & - & - & - & - & - & 120 \\
\hline Enchytronia sp. (TENU) & 120 & - & - & - & 160 & - \\
\hline Enchytronia pygmaea Graefe \& Schmelz, 2017 & & & & & & 100 \\
\hline Fridericia auritoides Schmelz, 2003 & - & - & - & - & - & 720 \\
\hline Fridericia benti Schmelz, 2002 & 60 & - & - & - & - & - \\
\hline Fridericia bisetosa (Levinsen, 1884) & 2980 & - & - & - & - & 1980 \\
\hline Fridericia bretscheri Southern, 1907 & - & - & - & - & - & 2460 \\
\hline Fridericia brunensis Schlaghamerský, 2008 & 320 & - & 30 & - & - & - \\
\hline Fridericia bulboides Nielsen \& Christensen, 1959 & 1220 & - & - & 180 & - & - \\
\hline Fridericia ciliotheca Schmelz \& Collado, 2013 & - & - & - & - & - & 460 \\
\hline Fridericia connata Bretscher, 1902 & 240 & - & 20 & - & - & 200 \\
\hline Fridericia cylindrica Springett, 1971 & - & - & - & - & - & 40 \\
\hline Fridericia dura (Eisen, 1879) & 620 & - & - & - & - & - \\
\hline Fridericia isseli Rota, 1994 & - & - & - & - & - & 340 \\
\hline Fridericia larix Schmelz \& Collado, 2005 & - & - & - & - & - & 160 \\
\hline Fridericia monochaeta Rota, 1995 & - & - & - & - & 800 & 300 \\
\hline Fridericia paroniana Issel, 1904 & - & - & - & - & - & 5520 \\
\hline Fridericia ratzeli (Eisen, 1872 ) & 740 & - & 20 & 60 & - & - \\
\hline Fridericia striata (Levinsen, 1884) & 280 & - & - & 90 & 3,120 & 1780 \\
\hline Fridericia tuberosa Rota, 1995 & - & - & - & - & - & 240 \\
\hline Fridericia sp. (PANO) & - & - & - & - & 1,080 & - \\
\hline Fridericia sp. (PERT & - & - & - & - & - & 1980 \\
\hline Marionina argentea (Michaelsen, 1889) & - & - & - & - & - & 40 \\
\hline Marionina clavate Nielsen \& Christensen, 1961 & - & 5100 & 5340 & 1160 & - & - \\
\hline Marionina filiformis Nielsen \& Christensen, 1959 & - & - & - & 60 & - & - \\
\hline
\end{tabular}




\begin{tabular}{|l|c|c|c|c|c|c|}
\hline Genus / Species & \multicolumn{2}{|c|}{$\begin{array}{c}\text { Saxony } \\
\text { (Germany) }\end{array}$} & \multicolumn{2}{c|}{ Hesse (Germany) } & \multicolumn{2}{c|}{ Portugal } \\
\hline & $\begin{array}{c}\text { Vier- } \\
\text { eichen }\end{array}$ & $\begin{array}{c}\text { Ullers- } \\
\text { dorf }\end{array}$ & $\begin{array}{c}\text { Rüssels- } \\
\text { heim }\end{array}$ & $\begin{array}{c}\text { Lam- } \\
\text { pertheim }\end{array}$ & Cerdeira & St. Olaia \\
\hline Marionina simillima Nielsen \& Christensen, 1959 & - & - & - & - & 5460 & - \\
\hline Marionina sp. (SIM?) & 20 & - & - & - & - & - \\
\hline Mesenchytraeus glandulosus(Levinsen, 1884) & 40 & - & - & - & - & - \\
\hline Mesenchytraeus pelicensis Issel, 1905 & 120 & 80 & 30 & - & - & - \\
\hline Oconnorella cambrensis(O'Connor, 1963) & - & 6680 & 310 & 7800 & - & - \\
\hline $\begin{array}{l}\text { Oconnorella tubifera } \text { (Nielsen \& Christensen, } \\
\text { 1959) }\end{array}$ & 14360 & 220 & - & 1400 & - & - \\
\hline Stercutus niveus Michaelsen, 1888 & - & 80 & - & - & - & - \\
\hline Abundance (Ind/m ${ }^{2}$ ) & $\mathbf{9 5 8 2 0} \pm$ & $\mathbf{4 6 7 6 0} \pm$ & $\mathbf{2 5 0 2 0} \pm$ & $\mathbf{4 8 7 3 5} \pm$ & $\mathbf{4 0 4 0 0} \pm$ & $\begin{array}{l}\mathbf{4 1 3 6 0} \pm \\
\mathbf{2 7 3 0 0}\end{array}$ \\
$\mathbf{2 9 4 7 0}$ & $\mathbf{1 1 2 3 2}$ & $\mathbf{2 6 3 2 4}$ & $\mathbf{2 6 5 4 5}$ & $\mathbf{2 6 4 8 3}$ \\
\hline Species number per site & $\mathbf{2 1}$ & $\mathbf{1 3}$ & $\mathbf{1 6}$ & $\mathbf{1 9}$ & $\mathbf{1 1}$ & $\mathbf{2 7}$ \\
\hline
\end{tabular}

*=Cognettia sphagnetorum auct. (partim). See Martinsson et al. (2015) for details. The valid genus name is controversial and awaits a ruling by the International Commission on Zoological Nomenclature.

species. Our species number range, 13-21 fits very well to this average richness. To our knowledge, no quantitative studies on enchytraeid abundance and community structure have been carried out at Portuguese forest sites in general so far.

Regarding the biogeographical distribution, it seems that, despite the large geographical distance between the German and Portuguese sites, there is considerable overlap in faunal composition, although the overlap is smaller than in the case of earthworms. The large number of previously known species in Portugal confirmed previous records, reviewed in Schmelz \& Collado (2013), who list altogether 32 species for Portugal. Two third of the species have also been recorded in Germany, thus most species belong to both the Portuguese and the German enchytraeid fauna. The high number of species found only in St. Olaia (18), including five species not yet described, indicates that the Portuguese enchytraeid fauna is far from being fully explored. In contrast to Central Europe, many new species were found not only in the species-rich genus Fridericia but also in genera such as Achaeta or Enchytronia. This is especially important when planning to use enchytraeids as indicators of soil quality (i.e. in terms of soil biodiversity). Species already known from other areas can be classified ecologically (e.g., several species found at Cerdeira such as $A$. affinis or E. norvegicus are known to be acidophilous).
Five of the six study sites have a $\mathrm{pH}<4$ and the sixth one, St. Olaia, is also acidic, although less so (Table 1), meaning that a relatively small number of acidophilous species (often in high numbers) are expected to occur there. However, it is not clear why at five out of six sites the species number is higher than expected. This may partly be explained by site-specific characteristics which, however, are difficult to identify. For example, in Lampertheim the vegetation pattern is very heterogeneous at a small scale, meaning that open spots and typical forest areas intermingle, providing different ecological niches at small scales. Rüsselsheim, on the other hand, is a forest located close to a densely populated city area, which - after a fire - had partly been afforested about 20 years ago. Thus, it can be considered to represent a disturbed site. However, while this stress may explain the low enchytraeid abundance, it seems to be contradictory to the relatively high species number.

The Cerdeira site is located in a mountainous region, often with shallow (i.e. less than $10 \mathrm{~cm}$ ) soils and steep hills. This means that soils regularly dry up, which may explain the overall low abundance in comparison to such forests in general. A preliminary sampling in summer with dustdry soil yielded no enchytraeids. Typical species of such acid sites are: Ac. affinoides, Co. cognettii, En. norvegicus and Fr. striata. New geographical elements (to be checked with more 
sites) are Fr. monochaeta, Fr. (PANO), and $M a$. simillima.

The forest of St. Olaia is the last remnant of a larger forest, being located on a small hill surrounded by agricultural land. The dominance of species from the genera Enchytraeus and Fridericia indicates neutral soils, which is interesting since the soil $\mathrm{pH}$ was measured as being just 5.0. In addition, the absence of Henlea species indicates dry conditions. New zoogeographical elements (to be checked ecologically with more sites) are represented by $F r$. auritoides, $F r$. bretscheri, Fr. ciliotheca, and Fr. monochaeta.

Finally, some special remarks on the taxonomy and biogeography regarding the two Portuguese sites can be made. At St. Olaia, Fr. ciliotheca, recently described from Coimbra (about $80 \mathrm{~km}$ to the west), was found for the first time following its original description. Fr. larix was found for the second time following its original description. Thus far it is only known from Ireland (original description) and England (unpublished). Possibly, it is a truly Atlantic species. The record of Enchytronia pygmaea at St.Olaia was included in the original description of the species (Graefe \& Schmelz 2017).

Acknowledgements - We thank all colleagues in Flörsheim, Görlitz and Coimbra who helped with the field samplings and the participants of the $12^{\text {th }}$ International Symposium on Enchytraeidae (Tihany, Hungary (27-29 June 2016)) for their contributions when discussing this issue. In addition, we would like to thank the referees for their very constructive remarks.

\section{REFERENCES}

Blankinship, J.C., Niklaus, P.A. \& Hungate, B.A. (2011): A meta-analysis of responses of soil biota to global change. Oecologia, 165: 553-5652. doi: $10.1007 / \mathrm{s} 00442-011-1909-0$

Blouin, M, Hodson, M.E., Delgado, E.A. BAKer, D., BrussaArD, L., ButT, K.R., Dai, J., DeNDOOVen, L., Peres, G., Tondoh, J.E., Cluzeau, D. \& BRUN, J.-J. BRUN (2013): A review of earthworm impact on soil function and ecosystem services. European Journal of Soil Science, 64: 161-182. doi: $10.1111 /$ ejss. 12025
BouchÉ, M. B. (1972): Lombriciens de France. Écologie et systéematique. INRA Publ. 72-2, Institut National de Recherches Agriculturelles Annales de Zoologie. Ecologie animale, hors série 72 (2). Paris, $671 \mathrm{pp}$.

Bussotti, F., Pollastrini, M., Holland, V. \& BRÜGGEMANN, W. (2015): Functional traits and adaptive capacity of European forests to climate change. Environmental and Experimental Botany, 111: 91-113. doi: 10.1016/j.envexpbot.2014.11.006

CsuZDI, Cs. \& ZICSI, A. (2003): Earthworms of Hungary (Annelida: Oligochaeta, Lumbricidae). Pedozoologica Hungarica No. 1. Hungarian Natural History Museum and Systematic Zoology Research Group of the Hungarian Academy of Sciences, Budapest. $271 \mathrm{pp}$.

Dorow, W.H.O., LANGE, A., QuerbaCH, A. \& BRÜGGEMANN, W. (2012): Mediterranean Oaks (Quercus spp.) and Central European Oak-feeding Lepidoptera in Southern Hesse. Entomology General, 34: 119-130. doi: 10.1127/entom.gen/34/2012/119

GRAEFE, U. \& SChMELZ, R. M. (2017): Description of Enchytronia pygmaea sp. n. (Enchytraeidae, Clitellata), a very small enchytraeid in European soils. Opuscula Zoologica (Budapest), 48(Suppl. 2): 310. doi: 10.18348/opzool.2017.S2.3

GRAFF, O. (1953): Die Regenwürmer Deutschlands. Schriftenreihe der Forschungsanstalt für Landwirtschaft Braunschweig-Völkenrode 7, Schaper. Germany, $81 \mathrm{pp}$.

ISO (INTERNATIONAL ORGANIZATION FOR STANDARDIZATION) (2006): Soil quality - Sampling of soil invertebrates Part 1: Hand-sorting and formalin extraction of earthworms. ISO 23611-1. Geneva, Switzerland.

ISO (INTERNATIONAL ORGANIZATION FOR STANDARDIZATION) (2007): Soil quality - Sampling of soil invertebrates Part 3: Sampling and soil extraction of enchytraeids. ISO 23611-3. Geneva, Switzerland.

JÄNSCH, S., STEFFENS, L., HÖFER, H., HORAK, F., ROßNickoll, M, Russell, D., ToschKI, A. \& RÖMBKE, J. (2013): State of knowledge of earthworm communities in German soils as a basis for biological soil quality assessment. Soil Organisms, 85: 215-232 + Electronic Supplement.

Martinsson, S., Rota, E. \& ErsÉUs, C. (2015): Revision of Cognettia (Clitellata, Enchytraeidae): 
reestablishment of Chamaedrilus and description of cryptic species in the sphagnetorum complex. Systematics and Biodiversity, 13: 257-277. doi: $10.1080 / 14772000.2014 .986555$

Peel, M.C., Finlayson, B.L. \& Mcmahon, T.A. (2007): Updated world map of the Koppen-Geiger climate classification. Hydrology and Earth System Sciences, 11: 1633-1644. doi: 10.5194/hess-11-1633-2007

Pflug, E. \& BRÜGgemann, W. (2012): Frost-acclimation of photosynthesis in overwintering Mediterranean holm oak, grown in Central Europe. International Journal of Plant Biology, 3:e1. doi: $10.4081 / \mathrm{pb} .2012 . \mathrm{el}$

RÖMBKE, J., JÄNSCH, S., HÖFER, H., HORAK, F., ROßNiCKOLL, M., RUSSELl, D. \& TOSCHKI, A. (2013): State of knowledge of enchytraeid communities in German soils as a basis for biological soil quality assessment. Soil Organisms 85: 123-146 + Electronic Supplement.

SChär, C., Vidale, P., LÜThI, D., Frei, C., HäBerLI, C., Liniger, M.A. \& APPENZELleR, C. (2004): The role of increasing temperature variability in European summer heatwaves. Nature, 427: 332335. doi: 10.1038 /nature 02300

SCHMELZ, R.M. \& COLLADO, R. (2010): A guide to European terrestrial and freshwater species of Enchytraeidae (Oligochaeta). Soil Organisms, 82: 1-176.

SchmelZ, R. M. \& Collado, R. (2012): An updated checklist of currently accepted species of Enchytaeidae (Oligochaeta). vTI Agriculture and Forestry Research, Special Issue 357: 67-87.
SCHMELZ, R. M. \& COLLADO, R. (2013): Enchytraeidae (Oligochaeta, Annelida) from a field site in Portugal, with the description of five new species and a redescription of Enchylea heteroducta Nielsen \& Christensen, 1963. Zootaxa 3647: 307328. doi: 10.11646/zootaxa.3647.2.4

Schmidl, J., Goodess, C.M., Frei, C., Haylock, M.R., HundeCHA, Y., RiBALAYGUA, J. \& SCHMITH, T. (2007): Statistical and dynamical downscaling of precipitation: An evaluation and comparison of scenarios for the European Alps. Journal of Geophysical Research - Atmosphere, 112: 19842012. doi: $10.1029 / 2005 J \mathrm{DD} 007026$

SIMS, R. W. \& GERARD, B.M. (1999): Earthworms. In. KERMACK, D.M. \& R. S. K. BARNES (eds.): Synopses of the British Fauna (New Series) No. 31. E. J. Brill / W. Backhuys, London. UK. 171 pp.

WaLl, D.H., BRAdFord, M.A., St. JOHN, M.G., Trofymow, J.A., Behan-Pelletier, V., Bignell, D.E., DANGERfield, J.M., PARTON, W.J., RUSEK, J., Voigt, W., Wolters, V., Zadeh Gardel, H., AYUKe, F.O., BAShFord, R., Beljakova, O.I., Bohlen, P.J., BRAuman, A., Flemming, S., HENSCHEL, J.R., JOHNSON, D.L., HEFIN JONES, T., Kovarova, M., KranabetTer, J.M., Kutny, L., Lin, K-C., Maryati, M., Masse, D., POKARZHEVSKII, A., RAHMAN, H., SABARA, M.G., SALAMON, J.-A., SWIFT, M.J., VARELA, A., VASCONCELOS, H.L., White, D. \& ZoU, X. (2008): Global decomposition experiment shows soil animal impacts on decomposition are climatedependent. Global Change Biology, 14: 26612677. doi: 10.1111/j.1365-2486.2008.01672.x 
